\title{
Criteria for the density property of complex manifolds ${ }^{\star}$
}

\section{Shulim Kaliman ${ }^{1}$, Frank Kutzschebauch ${ }^{2}$}

1 Department of Mathematics, University of Miami, Coral Gables, FL 33124, USA (e-mail: kaliman@math.miami .edu)

2 Mathematisches Institut, Universität Bern, Sidlerstr. 5, CH-3012 Bern, Switzerland (e-mail: Frank. Kutzschebauch@math.unibe.ch)

Oblatum 16-VIII-2006 \& 15-X-2007

Published online: 1 December 2007 - (C) Springer-Verlag 2007

\section{Introduction}

The ground-breaking papers of Andersén and Lempert [1,2] established remarkable properties of the automorphism group of $\mathbb{C}^{n}(n \geq 2)$ which imply, in particular, that any local holomorphic phase flow on a Runge domain $\Omega$ in $\mathbb{C}^{n}$ can be approximated by global holomorphic automorphisms of $\mathbb{C}^{n}$ (for an exact statement see Theorem 2.1 in [9]).

The next step in the development of the Andersén-Lempert theory was made by Varolin who extended it from Euclidean spaces to a wider class of algebraic complex manifolds. He realized also that the following density property is crucial for this theory.

1.1. Definition. A complex manifold $X$ has the density property if in the compact-open topology the Lie algebra $\operatorname{Lie}_{\text {hol }}(X)$ generated by completely integrable holomorphic vector fields on $X$ is dense in the Lie algebra $\mathrm{VF}_{\text {hol }}(X)$ of all holomorphic vector fields on $X$. An affine algebraic manifold $X$ has the algebraic density property if the Lie algebra $\operatorname{Lie}_{\text {alg }}(X)$ generated by completely integrable algebraic vector fields on it coincides with the Lie algebra $\operatorname{VF}_{\text {alg }}(X)$ of all algebraic vector fields on it (clearly, the algebraic density property implies the density property).

* This research was started during a visit of the second author to the University of Miami, Coral Gables, and continued during a visit of both of us to the Max Planck Institute of Mathematics in Bonn. We thank these institutions for their generous support and excellent working conditions. The research of the first author was also partially supported by NSA Grant no. H98230-06-1-0063 and the second one by Schweizerische Nationalfonds grant No 200021-107477/1.

Mathematics Subject Classification (2000): Primary: 32M05,14R20. Secondary: 32M05, $14 \mathrm{R} 20$ 
In this terminology the main observation of the Andersén-Lempert theory says that $\mathbb{C}^{n}(n \geq 2)$ has the algebraic density property. Varolin and Toth [16-18] established the density property for some manifolds including semisimple complex Lie groups and some homogenous spaces of semi-simple Lie groups. Their proof relies heavily on representation theory and does not, for example, lead to an answer in the case of other linear algebraic groups.

In this paper we suggest new effective criteria for the density property. This enables us to give a trivial proof of the original Andersén-Lempert result and to establish (almost free of charge) the algebraic density property for all linear algebraic groups whose connected components are different from tori or $\mathbb{C}_{+}$. As another application of this approach we tackle the question (asked among others by F. Forstnerič) about the density of algebraic vector fields on Euclidean space vanishing on a codimension 2 subvariety.

Our method of establishing the algebraic density property for an affine algebraic variety $X$ consists of two ingredients described in Sect. 2. First, we try to find a nontrivial $\mathbb{C}[X]$-module $L$ (over the algebra $\mathbb{C}[X]$ of regular functions on $X)$ in $\operatorname{Lie}_{a l g}(X)$. It turns out that this requires the existence of two commuting completely integrable algebraic vector fields on $X$ satisfying some compatibility condition (see, Definition 2.5 below). Second, since $\operatorname{Lie}_{\text {alg }}(X)$ is invariant under algebraic automorphisms of $X$, in the presence of some homogeneity property of $X$ we can increase $L$ so that it coincides with the $\mathbb{C}[X]$-module of all algebraic vector fields (in which $L$ is contained, of course, as a submodule). In Sects. 3 and 4 we develop technique for checking this compatibility condition and apply it in the cases of linear algebraic groups and the complements to codimension 2 subvarieties in Euclidean spaces.

Acknowledgements. We would like to thank D. Akhiezer for inspiring discussions and consultations, F. Donzelli for catching some inaccuracies, and the referee for valuable comments that lead, in particular, to the present formulation of Proposition 3.9.

\section{New approach to the Andersén-Lempert theory}

The homogeneity property mentioned before is reflected in the following.

2.1. Definition. Let $X$ be an algebraic manifold and $x_{0} \in X$. A finite subset $M$ of the tangent space $T_{x_{0}} X$ is called a generating set if the image of $M$ under the action of the isotropy subgroup of $x_{0}$ (in the group of all algebraic automorphisms Aut $X$ of $X$ ) generates the whole space $T_{x_{0}} X$.

The manifold $X$ will be called tangentially semi-homogeneous if it is homogeneous (with respect to Aut $X$ ) and admits a generating set consisting of one vector.

Theorem 1. Let $X$ be a homogeneous (with respect to Aut $X$ ) affine algebraic manifold with algebra of regular functions $\mathbb{C}[X]$, and $L$ be a submodule of the $\mathbb{C}[X]$-module of all vector fields such that $L \subset \operatorname{Lie}_{\mathrm{alg}}(X)$. Suppose 
that the fiber of $L$ over some $x_{0} \in X$ contains a generating set. Then $X$ has the algebraic density property.

Proof. The $\mathbb{C}[X]$-modules $T X$ and $L$ generate coherent sheaves $\mathcal{T}$ and $\mathcal{L}$ on $X$ where $\mathcal{L}$ is a subsheaf of $\mathcal{T}$. The action of $\alpha \in$ Aut $X$ maps $\mathcal{L}$ onto another coherent subsheaf $\mathcal{L}_{\alpha}$ of $\mathcal{T}$. The sum of such subsheaves with $\alpha$ running over a finite subset of Aut $X$ is a coherent subsheaf $\mathcal{E}$ of $\mathcal{T}$. Let $\mathfrak{m}$ be the maximal ideal for $x_{0}$. Definition 2.1 implies that $\mathcal{E}$ can be chosen so that $\mathscr{E} / \mathfrak{m} \mathscr{E}$ coincides with $T_{x_{0}} X$. Furthermore, since $X$ is homogeneous we can suppose that this is true for every point in $X$. Thus $\mathscr{E}=\mathcal{T}$ [12, Chap. II, Exercise 5.8]. Since composition with automorphisms preserves complete integrability, all global sections of $\mathscr{E}$ are in $\operatorname{Lie}_{\text {alg }}(X)$ which concludes the proof.

Another ingredient of our method is rooted in a new proof of the following fact.

2.2. Corollary (The main observation of the Andersén-Lempert theory). For $n \geq 2$ the space $\mathbb{C}^{n}$ has the algebraic density property.

Proof. Let $x_{1}, \ldots, x_{n}$ be a coordinate system on $\mathbb{C}^{n}$ and $\delta_{i}=\partial / \partial x_{i}$ be the partial derivative, i.e. $\operatorname{Ker} \delta_{i}$ is the ring of polynomials independent of $x_{i}$. Hence the polynomial ring $\mathbb{C}^{[n]}$ is generated as a vector space by elements of $\operatorname{Ker} \delta_{1} \cdot \operatorname{Ker} \delta_{2}$. Note also that for $f_{i} \in \operatorname{Ker} \delta_{i}$ the algebraic vector fields $f_{i} \delta_{i}$ and $x_{i} f_{i} \delta_{i}$ are completely integrable. Then the field

$$
\left[f_{1} \delta_{1}, x_{1} f_{2} \delta_{2}\right]-\left[x_{1} f_{1} \delta_{1}, f_{2} \delta_{2}\right]=f_{1} f_{2} \delta_{2}
$$

belongs to $\operatorname{Lie}_{\text {alg }}(X)$ since $x_{1} f_{2} \in \operatorname{Ker} \delta_{2}$. Thus $\operatorname{Lie}_{\text {alg }}(X)$ contains all algebraic fields proportional to $\delta_{2}$. Since $\mathbb{C}^{n}$ is clearly tangentially semihomogeneous Theorem 1 implies the desired conclusion.

2.3. Remark. There is no need to use tangential semi-homogeneity in this proof since we can replace $\delta_{2}$ by any other partial derivative $\delta_{i}$ and obtain each algebraic vector field as a sum of fields proportional to $\delta_{i}, i=1, \ldots, n$. However there are no partial derivatives on affine algebraic varieties different from Euclidean spaces. Hence formalization of this argument requires Theorem 1 and some substitution for partial derivatives.

We remind that for a completely integrable algebraic vector field on an affine algebraic variety its phase flow is only a holomorphic $\mathbb{C}_{+}$-action that is not necessarily algebraic.

2.4. Definition. An algebraic vector field $\delta$ on $X$ is called semi-simple if its phase flow generates an algebraic $\mathbb{C}^{*}$-action on $X$. A vector field $\sigma$ is called locally nilpotent if its phase flow is an algebraic $\mathbb{C}_{+}$-action on $X$. In the last case $\sigma$ can be viewed as a locally nilpotent derivation on the algebra $\mathbb{C}[X]$ of regular functions on $X$. That is, for every $f \in \mathbb{C}[X]$ there is $n=n(f)$ for which $\sigma^{n}(f)=0$. 
There is one-to-one correspondence between the set of locally nilpotent derivations on $\mathbb{C}[X]$ and the set of algebraic $\mathbb{C}_{+}$-actions on $X$ (e.g., see [6] for details). We shall often use below the fact that for any locally nilpotent derivation $\sigma$ and a regular function $f$ from its kernel $\operatorname{Ker} \sigma$ (resp. regular function $f$ of degree 1 with respect to $\sigma$, i.e. $\sigma(f) \in \operatorname{Ker} \sigma \backslash 0)$ the vector field $f \sigma$ is locally nilpotent (resp. completely integrable).

2.5. Definition. Let $\delta_{1}$ and $\delta_{2}$ be nontrivial algebraic vector fields on an affine algebraic manifold $X$ such that $\delta_{1}$ is a locally nilpotent derivation on $\mathbb{C}[X]$, and $\delta_{2}$ is either also locally nilpotent or semi-simple. That is, $\delta_{i}$ generates an algebraic action of $H_{i}$ on $X$ where $H_{1} \simeq \mathbb{C}_{+}$and $H_{2}$ is either $\mathbb{C}_{+}$or $\mathbb{C}^{*}$. We say that $\delta_{1}$ and $\delta_{2}$ are compatible if

(i) the vector space $\operatorname{Span}\left(\operatorname{Ker} \delta_{1} \cdot \operatorname{Ker} \delta_{2}\right)$ generated by elements from $\operatorname{Ker} \delta_{1} \cdot \operatorname{Ker} \delta_{2}$ contains a non-zero ideal in $\mathbb{C}[X]$ and

(ii) some element $a \in \operatorname{Ker} \delta_{2}$ is of degree 1 with respect to $\delta_{1}$, i.e. $\delta_{1}(a) \in$ $\operatorname{Ker} \delta_{1} \backslash\{0\}$.

2.6. Remark. Instead of Condition (ii) suppose now that $\delta_{1}$ and $\delta_{2}$ commute. Then by Corollary 3.5 below Condition (i) implies that the $H_{1}$-action on $X$ generates a nontrivial algebraic $\mathbb{C}_{+}$-action on $X / / H_{2}$. Taking an element of $\mathbb{C}\left[X / / H_{2}\right]$ whose degree with respect to this action is 1 we can treat its lift-up to $X$ as $a$. That is, for such commutative $\delta_{1}$ and $\delta_{2}$ Condition (ii) is automatic.

Theorem 2. Let $X$ be a smooth homogeneous affine algebraic manifold with finitely many pairs of compatible vector fields $\left\{\delta_{1}^{k}, \delta_{2}^{k}\right\}_{k=1}^{m}$ such that for some point $x_{0} \in X$ vectors $\left\{\delta_{2}^{k}\left(x_{0}\right)\right\}_{k=1}^{m}$ form a generating set. Then $\operatorname{Lie}_{\text {alg }}(X)$ contains a nontrivial $\mathbb{C}[X]$-module and $X$ has the algebraic density property.

Proof. Let $\delta_{1}$ and $\delta_{2}$ be one of our pairs. Choose an element $a \in \operatorname{Ker} \delta_{2}$ of degree 1 with respect to $\delta_{1}$ and set $b=\delta_{1}(a)$. Let $f_{i} \in \operatorname{Ker} \delta_{i}$. Then $\left[a f_{1} \delta_{1}, f_{2} \delta_{2}\right]-\left[f_{1} \delta_{1}, a f_{2} \delta_{2}\right]=-b f_{1} f_{2} \delta_{2}$. The last vector field is from $\mathrm{Lie}_{\text {alg }}(X)$ and since $\delta_{1}$ and $\delta_{2}$ are compatible, Definition 2.5 implies that sums of such vector fields include every vector field of form $I \delta_{2}$ where $I$ is a non-zero ideal in $\mathbb{C}[X]$. Applying this argument to all compatible pairs we see that $\operatorname{Lie}_{\text {alg }}(X)$ contains all linear combinations of $\delta_{2}^{k}$ with coefficients in some non-zero ideal $J \subset \mathbb{C}[X]$. Since under a small perturbation of $x_{0}$ the set $\left\{\delta_{2}^{k}\left(x_{0}\right)\right\}_{k=1}^{m}$ remains a generating set we can suppose that $x_{0}$ does not belong to the zero locus of $J$. Hence by Theorem $1 X$ has the algebraic density property.

2.7. Remark. If $X$ is tangentially semi-homogenous and, furthermore, any non-zero tangent vector (at any point) is a generating set, then Theorem 2 implies that for the algebraic density property a single pair of compatible vector fields is enough. The assumption that $\delta_{2}$ is locally nilpotent or semisimple is not used in the above proof. It is sufficient that $\delta_{2}$ is completely integrable. 
2.8. Corollary. Let $X_{1}$ and $X_{2}$ be homogeneous affine algebraic manifolds such that each $X_{i}$ admits a finite number of completely integrable algebraic vector fields $\left\{\delta_{i}^{k}\right\}_{k=1}^{m_{i}}$ whose values at some point $x_{i} \in X_{i}$ form a generating set and, furthermore, in the case of $X_{1}$ these vector fields are locally nilpotent. Then $X_{1} \times X_{2}$ has the algebraic density property.

Proof. Note that $\delta_{1}^{k}$ and $\delta_{2}^{j}$ generate compatible vector fields on $X_{1} \times X_{2}$ which we denote by the same symbols. Applying isotropy groups one can suppose that $\left\{\delta_{i}^{k}\left(x_{i}\right)\right\}$ is a basis of $T_{x_{i}} X_{i}$. In order to show that the set of vectors $M=\left\{0 \times \delta_{2}^{k}\left(x_{2}\right)\right\}$ form a generating set in $T_{x_{1} \times x_{2}}\left(X_{1} \times X_{2}\right)$ we need the following fact that is obvious in a local coordinate system.

Claim. Let $X$ be a complex manifold and let $v$ be a vector field on $X$. Suppose that $f$ is a holomorphic function from Ker $v$ and $x_{0} \in f^{-1}(0)$. Then phase flow induced by the vector field $f v$ generates a linear action on the tangent space $T_{x_{0}} X$ given by the formula $w \rightarrow w+d f(w) v\left(x_{0}\right)$ where $d f$ is the differential and $w \in T_{x_{0}} X$. In particular, the span of the orbit of $w$ under this phase flow contains vector $d f(w) v\left(x_{0}\right)$.

Applying this claim for $v=\delta_{1}^{j}$ we see that the orbit of $M$ under the isotropy group of $x_{1} \times x_{2}$ contains all vectors of form $\delta_{1}^{j}\left(x_{1}\right) \times \delta_{2}^{k}\left(x_{2}\right)$. Thus $M$ is a generating set and we are done by Theorem 2 .

2.9. Remark. The reason why we use the locally nilpotent $\delta_{1}^{j}$ in this proof as $v$ and not (the possibly semi-simple) $\delta_{2}^{j}$ is the following: The vector field $f \delta_{2}^{j}$ with $f \in \operatorname{Ker} \delta_{2}^{j}$ may not generate an algebraic action while $f \delta_{1}^{j}$ with $f \in \operatorname{Ker} \delta_{1}^{j}$ always generates an algebraic action. It is worth mentioning if one wants to prove density property instead of algebraic density property the use of $\delta_{2}^{j}$ is permissible.

2.10. Example. (1) Let $X=\mathbb{C}^{k} \times\left(\mathbb{C}^{*}\right)^{l}$ with $k \geq 1$ and $k+l \geq 2$. Then $X$ has algebraic density property by Corollary 2.8 .

(2) If $G$ is a simple Lie group then it is tangentially semi-homogeneous since the adjoint action of $G$ generates an irreducible representation on the tangent space $\mathfrak{g}$ at the identity $e$ (i.e., any non-zero vector in $T_{e} G$ is a generating set). Let $X$ be $S L_{n}(\mathbb{C})$ with $n \geq 2$, i.e. $X$ is tangentially semihomogeneous. Then every $x \in X$ is a matrix $\left(c_{k j}\right)$ with determinant 1 . Set $\delta_{1}\left(c_{1 j}\right)=c_{n j}$ and $\delta_{1}\left(c_{k j}\right)=0$ for $k \neq 1$. Set $\delta_{2}\left(c_{n j}\right)=c_{1 j}$ and $\delta_{2}\left(c_{k j}\right)=0$ for $k \neq n$. Note that constants and functions depending on $c_{k j}, k \neq 1$ are in $\operatorname{Ker} \delta_{1}$ while constants and functions depending on $c_{k j}, k \neq n$ are in $\delta$. Therefore, Condition (i) of Definition 2.5 holds. Taking $c_{11}$ as $a$ in Condition (ii) we see that $\delta_{1}$ and $\delta_{2}$ are compatible. Thus $S L_{n}(\mathbb{C})$ has the algebraic density property.

\section{Density of affine algebraic groups with connected components different from tori or $\mathbb{C}_{+}$}

We start with a digest of the notion of categorical (algebraic) quotient and its properties which will be used extensively in the rest of this section. 
3.1. Definition. Let $G$ be a linear algebraic group acting algebraically on an affine algebraic variety $X$ and, therefore, on its algebra $\mathbb{C}[X]$ of regular functions (we are going to use this notation for the algebra of regular functions further for any variety $X$, not necessarily affine). Consider the subalgebra $\mathbb{C}[X]^{G}$ of $G$-invariant functions. Its spectrum is called the categorical quotient of this action and it is denoted by $X / / G$. The monomorphism $\mathbb{C}[X]^{G} \hookrightarrow \mathbb{C}[X]$ generates a dominant (but not necessarily surjective) morphism $\rho: X \rightarrow X / / G$ which is called the quotient morphism. The universal property of categorical quotients says that any morphism from $X$ that is constant on orbits of $G$ factors through $\rho$.

3.2. Remark. For a reductive $G$ the subalgebra $\mathbb{C}[X]^{G}$ is always finitely generated by Nagata's theorem and, therefore, $X / / G$ can be viewed as an affine algebraic variety. Furthermore, $\rho$ is surjective in this case, the points of $X / / G$ are in one-to-one correspondence with closed orbits of $G$ in $X$, and every fiber of $\rho$ is the union of those orbits whose closure contains the corresponding closed orbit (e.g., see [15]). In particular, if each orbit is closed then the categorical quotient coincides with the usual geometric quotient (this happens, say, when a reductive subgroup acts on a linear algebraic group by multiplication). If $G$ is not reductive then $\mathbb{C}[X]^{G}$ is not finitely generated in general (by Nagata's counterexample to the fourteenth Hilbert problem). However, $X / / G$ can be viewed as a quasi-affine algebraic variety and $\mathbb{C}[X]^{G}$ as its algebra of regular functions [21]. We will work mostly with $G \simeq \mathbb{C}_{+}$. In this case general fibers of $\rho$ are always orbits of the $\mathbb{C}_{+}$-action (i.e. $\operatorname{dim} X / / G=\operatorname{dim} X-1$ ) and $\mathbb{C}[X]^{G}$ coincides with the kernel of the corresponding locally nilpotent derivation (e.g., see [6]).

3.3. Notation. In this section $H_{1}$ is isomorphic to $\mathbb{C}_{+}$and $H_{2}$ is isomorphic either to $\mathbb{C}_{+}$or $\mathbb{C}^{*}$. We suppose also that $X$ is a normal affine algebraic variety equipped with nontrivial algebraic $H_{i}$-actions where $i=1,2$ (in particular, each $H_{i}$ generates an algebraic vector field $\delta_{i}$ on $X$ ). The categorical quotients will be denoted $X_{i}=X / / H_{i}$ and the quotient morphisms by $\rho_{i}: X \rightarrow X_{i}$.

We start with a geometric reformulation of Definition 2.5.

3.4. Proposition. Set $\rho=\left(\rho_{1}, \rho_{2}\right): X \rightarrow Y:=X_{1} \times X_{2}$ and $Z$ equal to the closure of $\rho(X)$ in $Y$. Then $\operatorname{Span}\left(\operatorname{Ker} \delta_{1} \cdot \operatorname{Ker} \delta_{2}\right)$ contains a non-zero ideal of $\mathbb{C}[X]$ iff $\rho: X \rightarrow Z$ is a finite birational morphism.

Proof. Every non-zero element of $\operatorname{Span}\left(\operatorname{Ker} \delta_{1} \cdot \operatorname{Ker} \delta_{2}\right)$ is of the form $g \circ \rho$ where $g \in \mathbb{C}[Z]=\left.\mathbb{C}[Y]\right|_{Z}$. Thus $\operatorname{Span}\left(\operatorname{Ker} \delta_{1} \cdot \operatorname{Ker} \delta_{2}\right)$ coincides with the subalgebra $\rho^{*}(\mathbb{C}[Z]) \subset \mathbb{C}[X]$ and we need to establish when $\rho^{*}(\mathbb{C}[Z])$ contains a nontrivial ideal of $\mathbb{C}[X]$. Note that functions from any nontrivial ideal separate general points of $X$ while functions from $\rho^{*}(\mathbb{C}[Z])$ do not separate points of $\rho^{-1}(z)$ for any $z \in Z$. Hence $\rho: X \rightarrow Z$ must be birational if we want $\delta_{1}$ and $\delta_{2}$ to be compatible. 
Assume now that the closure of $Z \backslash \rho(X)$ contains a divisor $D \subset Z$. There is a rational function $f$ on $Z$ so that it has poles on $D$ and nowhere else. Multiplying $f$ by $h \in \mathbb{C}[Z]$ such that $h$ is not identically zero on $D$ but vanishes on $D \cap \rho(X)$ with sufficient multiplicity, one can suppose that $f \circ \rho$ is regular on $X$. On the other hand for $n$ sufficiently large and $g$ as before $g f^{n}$ has poles on $D$ and cannot be a regular function on $Z$. Thus $\left(g f^{n}\right) \circ \rho \notin \operatorname{Span}\left(\operatorname{Ker} \delta_{1} \cdot \operatorname{Ker} \delta_{2}\right)$ and the last vector space cannot contain a non-zero ideal in this case.

Let $\rho=v \circ \rho_{0}$ where $v: Z_{0} \rightarrow Z$ is a normalization of $Z$ and $\rho_{0}:$ $X \rightarrow Z_{0}$ is the induced morphism of normal varieties. Then $\rho_{0}$ is birational and $Z_{0} \backslash \rho_{0}(X)$ is of codimension at least 2 since otherwise even $\rho_{0}^{*}\left(\mathbb{C}\left[Z_{0}\right]\right)$ does not contain a nontrivial ideal of $\mathbb{C}[X]$. The indeterminacy set $V \subset$ $\rho_{0}(X)$ of the rational map $\rho_{0}^{-1}$ is of codimension at least 2 . Hence any regular function on $\rho_{0}(X) \backslash V$ extends to $Z_{0}$ by the Hartogs theorem. This implies that $\rho_{0}^{-1}$ is regular and, therefore, $\rho_{0}$ is an isomorphism, i.e. $\rho$ is finite birational. Since $v$ is finite $\mathbb{C}\left[Z_{0}\right]$ is generated over $\mathbb{C}[Z]$ by a finite number of functions of form $f_{i} / g_{i}, i=1,2, \ldots, n$ where $f_{i}$ and $g_{i}$ are regular on $Z$. Treat $\mathbb{C}[Z] \simeq v^{*}(\mathbb{C}[Z])$ as a subalgebra of $\mathbb{C}\left[Z_{0}\right]$ and consider the principal ideal $J$ in $\mathbb{C}\left[Z_{0}\right]$ generated by $\prod_{i=1}^{n} g_{i}$. By construction, $J \subset \mathbb{C}[Z]$. Hence $\rho^{*}(\mathbb{C}[Z])$ contains a non-zero ideal of $\mathbb{C}[X] \simeq \mathbb{C}\left[Z_{0}\right]$ which is the desired conclusion.

Note that for every (resp. a general) $\left(x_{1}, x_{2}\right) \in Z$ the set $\rho^{-1}\left(x_{1}, x_{2}\right)=$ $\rho_{1}^{-1}\left(x_{1}\right) \cap \rho_{2}^{-1}\left(x_{2}\right)$ is finite (resp. a singleton) in this Proposition. Hence a non-constant orbit of $H_{1}$ cannot be contained in a fiber of $\rho_{2}$ and we have the following.

3.5. Corollary. In the case of $\left[\delta_{1}, \delta_{2}\right]=0$ the $H_{1}$-action on $X$ generates a nontrivial $\mathbb{C}_{+}$-action on $X_{2}$.

3.6. Lemma. Suppose that $X, H_{i}, X_{i}, \delta_{i}$, and $\rho_{i}$ are as in Notation 3.3, and either $(i)\left[\delta_{1}, \delta_{2}\right]=0$; or (ii) $\delta_{1}$ and $\delta_{2}$ are both locally nilpotent and generate a Lie algebra $\mathfrak{s l}_{2}$ that induces an algebraic action of $S L_{2}(\mathbb{C})$ on $X$. Set $\Gamma=H_{1} \times H_{2}$ in Case (i), and $\Gamma=S L_{2}(\mathbb{C})$ in Case (ii). Suppose that $X^{\prime}$ is a normal affine algebraic variety equipped with a non-degenerate $\Gamma$-action and $p: X \rightarrow X^{\prime}$ is a finite $\Gamma$-equivariant morphism (for each $i=1,2)$, i.e. we have commutative diagrams

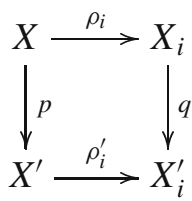

where $\rho_{i}^{\prime}: X^{\prime} \rightarrow X_{i}^{\prime}=X^{\prime} / / H_{i}$ is the quotient morphism of the $H_{i}$-action on $X^{\prime}$ (i.e., we treat $\mathbb{C}\left[X_{i}^{\prime}\right]$ as a subalgebra of $\left.\mathbb{C}\left[X^{\prime}\right]\right)$. Let $\operatorname{Span}\left(\mathbb{C}\left[X_{1}\right]\right.$. $\left.\mathbb{C}\left[X_{2}\right]\right)$ contain a non-zero ideal of $\mathbb{C}[X]$. Then $\operatorname{Span}\left(\mathbb{C}\left[X_{1}^{\prime}\right] \cdot \mathbb{C}\left[X_{2}^{\prime}\right]\right)$ contains a non-zero ideal of $\mathbb{C}\left[X^{\prime}\right]$. 
Proof. Since $p$ is finite, every $f \in \mathbb{C}\left[X_{i}\right] \subset \mathbb{C}[X]$ is a root of a minimal monic polynomial with coefficients in $\mathbb{C}\left[X^{\prime}\right]$ that are constant on $H_{i}$-orbits (since otherwise $f$ is not constant on these orbits). By the universal property these coefficients are regular on $X_{i}^{\prime}$, i.e. $f$ is integral over $\mathbb{C}\left[X_{i}^{\prime}\right]$ and $q_{i}$ is finite. Consider the commutative diagram

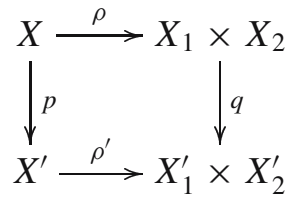

where $\rho=\left(\rho_{1}, \rho_{2}\right), \rho^{\prime}=\left(\rho_{1}^{\prime}, \rho_{2}^{\prime}\right)$, and $q=\left(q_{1}, q_{2}\right)$. Let $Z$ (resp. $\left.Z^{\prime}\right)$ be the closure of $\rho(X)$ in $X_{1} \times X_{2}$ (resp. $\rho^{\prime}\left(X^{\prime}\right)$ in $\left.X_{1}^{\prime} \times X_{2}^{\prime}\right)$. By Proposition 3.4 $\rho(X)=Z$ and, therefore, (since $q$ is finite) $q(\rho(X))=\rho^{\prime}\left(X^{\prime}\right)=Z^{\prime}$. Let $v: Z_{0} \rightarrow Z$ be a normalization, i.e. $X$ is naturally isomorphic to $Z_{0}$. Since $q \circ v: Z_{0} \rightarrow Z^{\prime}$ is finite it generates a finite morphism $X \simeq Z_{0} \rightarrow Z_{0}^{\prime}$ onto a normalization $Z_{0}^{\prime}$ of $Z^{\prime}$. The commutativity of our diagram implies that $\rho^{\prime}$ generates a finite morphism $\rho_{0}^{\prime}: X^{\prime} \rightarrow Z_{0}^{\prime}$. Thus it suffices to prove the following.

Claim. In the last commutative diagram of $\Gamma$-equivariant morphisms the fact that $\rho: X \rightarrow Z$ is birational and $p$ (and, therefore, $q$ ) is finite implies that morphism $\rho^{\prime}: X^{\prime} \rightarrow Z^{\prime}$ is birational.

For any $x \in X$ we set $x^{\prime}=p(x), x_{j}=\rho_{j}(x)$, and $x_{j}^{\prime}=q_{j}\left(x_{j}\right)=\rho_{j}^{\prime}\left(x^{\prime}\right)$. Assume that $x$ is a general point of $X$ and $y \in X$ is such that $\rho^{\prime}\left(x^{\prime}\right)=$ $\left(x_{1}^{\prime}, x_{2}^{\prime}\right)=\left(y_{1}^{\prime}, y_{2}^{\prime}\right)=\rho^{\prime}\left(y^{\prime}\right)$. Hence $q_{j}^{-1}\left(x_{j}^{\prime}\right)=q_{j}^{-1}\left(y_{j}^{\prime}\right)$ for $j=1,2$. Since $p$ is finite and $x^{\prime} \in X^{\prime}$ is a general point we have $\rho_{j}\left(p^{-1}\left(x^{\prime}\right)\right)=$ $q_{j}^{-1}\left(x_{j}^{\prime}\right)$ (otherwise $\rho_{j}$ is not dominant). Replacing $x$, if necessary, by another point from $p^{-1}\left(x^{\prime}\right)$, we can suppose that $x_{1}=y_{1}$ and that $y_{2}=z_{2}$ for some $z \in p^{-1}\left(x^{\prime}\right)$ (this means that $y$ and $x$ belong to the same orbit $O$ of $H_{1}$ because $x$ is general, see Remark 3.2). Since $x^{\prime}=z^{\prime}$ we have $x_{i}^{\prime}=y_{i}^{\prime}=z_{i}^{\prime}$ which implies that $q_{2}$ sends $x_{2}$ and $z_{2}$ to the same point. By the assumption $q_{2}$ is $H_{1}$-equivariant in Case (i). In particular, it sends the orbit $O_{2}=\rho_{2}(O) \subset$ $X_{2}$ into an $H_{1}$-orbit $O_{2}^{\prime} \subset X_{2}^{\prime}$. Both orbits are isomorphic to $H_{1} \simeq \mathbb{C}_{+}$, i.e. the $H_{1}$-equivariant morphism $\left.q_{2}\right|_{O_{2}}: O_{2} \rightarrow O_{2}^{\prime}$ must be an isomorphism. That is, $x_{2}=z_{2}=y_{2}$ and, therefore, $\rho(x)=\left(x_{1}, x_{2}\right)=\left(y_{1}, y_{2}\right)=\rho(y)$. Since $\rho: X \rightarrow Z$ is birational and $x$ is general we have $x=y$. Hence $\rho^{\prime}$ is an embedding in a neighborhood of a general point $x^{\prime}$ which implies the desired conclusion for (i).

In Case (ii) the general $\Gamma$-orbit $U$ in $X$ containing $O$ (resp. $\Gamma$-orbit $U^{\prime}=p(U) \subset X^{\prime}$ containing $O^{\prime}=p(O)$ ) is the set of left cosets $S L_{2}(\mathbb{C}) / K$ of a finite subgroup $K\left(\right.$ resp. $\left.K^{\prime}\right)$ in $S L_{2}(\mathbb{C})$. The $S L_{2}(\mathbb{C})$-action is generated by multiplication on the left while the $K$-action on $S L_{2}(\mathbb{C})$ is given by multiplication on the right. Hence the action of $\mathbb{C}_{+} \simeq H_{i}<S L_{2}(\mathbb{C})$ commutes with the $K$-action. This implies that each nonidentical element of $K$ sends 
any $H_{i}$-orbit isomorphically into a different orbit because among finite group actions only the trivial one commutes with the translation on $\mathbb{C}$. Thus the quotient morphism $S L_{2}(\mathbb{C}) \rightarrow U$ (resp. $S L_{2}(\mathbb{C}) \rightarrow U^{\prime}$ ) maps any $H_{i}$-orbit into a similar orbit isomorphically which implies that $\left.p\right|_{o}: O \rightarrow O^{\prime}$ is an isomorphism.

If $K$ is trivial one can suppose that the restrictions of $\delta_{1}$ and $\delta_{2}$ to $U \simeq S L_{2}(\mathbb{C})$ are as in Example 2.10(2). Then the quotient morphism $\varrho: S L_{2}(\mathbb{C}) \rightarrow S L_{2}(\mathbb{C}) / / H_{2} \simeq \mathbb{C}^{2}$ sends matrix $\left(c_{i j}\right) \in S L_{2}(\mathbb{C})$ into vector $\left(c_{11}, c_{12}\right)$, and $\left.\rho_{2}\right|_{U}=v \circ \varrho$ by the universal property of quotient morphisms. Since general fibers of $\varrho$ and $\rho_{2}$ are $H_{2}$-orbits, morphism $v: S L_{2}(\mathbb{C}) / / H_{2} \rightarrow$ $\rho_{2}(U)$ must be birational. The $H_{1}$-orbit $O$ of $x=\left(c_{i j}^{o}\right) \in S L_{2}(\mathbb{C})$ consists of matrices $\left(c_{i j}(t)\right)$ with $c_{1 j}(t)=c_{1 j}^{o}+t c_{2 j}^{o}$ and $c_{2 j}(t)=c_{2 j}^{o}$. Thus $\left.\varrho\right|_{o}: O \rightarrow$ $\varrho(O)$ is an isomorphism, and $x$ and $y$ belong to a Zariski open subset of $O$ on which $\left.\rho_{2}\right|_{O}$ is an embedding, because these points are general (in $X$ and, therefore, in $O$ ) and $v$ is birational. Similarly, if $K^{\prime}$ is trivial point $x^{\prime}$ belongs to a Zariski open subset of $O^{\prime}$ on which $\left.\rho_{2}^{\prime}\right|_{O^{\prime}}$ is an embedding. Taking into account the commutative diagram and the fact that $\left.p\right|_{o}$ is an isomorphism we see that $x_{2}$ and $z_{2}=y_{2}=\rho_{2}(y)$ are contained a Zariski open subset of $O_{2}=\rho_{2}(O)$ where $\left.q_{2}\right|_{O_{2}}$ is an embedding. The same argument as in Case (i) implies now that $\rho^{\prime}$ is birational.

In the general case multiplication of $S L_{2}(\mathbb{C})$ by elements of $K$ on the right generates a linear $K$-action on $\mathbb{C}^{2} \simeq \varrho\left(S L_{2}(\mathbb{C})\right)$ and we have morphism $\tau: U=S L_{2}(\mathbb{C}) / K \rightarrow \mathbb{C}^{2} / K$ generated by $\varrho$. Since $\left.\varrho\right|_{O}$ is an isomorphism and $\varrho(O)$ is a general line in $\mathbb{C}^{2}$ morphism $\left.\tau\right|_{O}: O \rightarrow \tau(O)$ is finite birational. As in the case of trivial $K$ we have $\left.\rho_{2}\right|_{U}=\mu \circ \tau$ where $\mu: \mathbb{C}^{2} / K \rightarrow \rho_{2}(U)$ is also birational. Thus the general points $x$ and $y$ belong again to a Zariski open subset of $O$ on which $\left.\rho_{2}\right|_{O}$ is an embedding. Similarly, point $x^{\prime}$ belongs to a Zariski open subset of $O^{\prime}$ on which $\left.\rho_{2}^{\prime}\right|_{O^{\prime}}$ is an embedding. Now the same argument as before concludes the proof.

Though Lemma 3.6 (together with a weak version of Corollary 3.10 that follows from it) enables us to go directly to the proof of Theorem 3, we include some other results to provide a stronger tool for establishing compatibility condition.

3.7. Lemma. Let the assumption of Lemma 3.6 hold with one exception: instead of the finiteness of $p$ we suppose that there are a surjective quasifinite morphism $r: S \rightarrow S^{\prime}$ of normal affine algebraic varieties equipped with trivial $\Gamma$-actions and a surjective $\Gamma$-equivariant morphism $\varrho^{\prime}: X^{\prime} \rightarrow S^{\prime}$ such that $X$ is isomorphic to fibred product $X^{\prime} \times_{S^{\prime}} S$ with $p: X \rightarrow X^{\prime}$ being the natural projection (i.e. $p$ is surjective quasi-finite). Then the conclusion of Lemma 3.6 remains valid.

Proof. By the Noether normalization theorem, taking the spectrum of the integral closure of $\mathbb{C}\left[S^{\prime}\right]$ in the field of rational functions on $S$, we obtain a normal affine algebraic variety $\tilde{S} \supset S$ with a finite morphism $\tilde{r}: \tilde{S} \rightarrow S^{\prime}$ extending $r: S \rightarrow S^{\prime}$. Set $\tilde{X}=X^{\prime} \times_{S^{\prime}} \tilde{S}$ and denote by $\tilde{p}: \tilde{X} \rightarrow X^{\prime}$ the 
natural projection. Then $\tilde{X}$ contains $X$ as a Zariski dense open subset, $\tilde{p}$ extends $p$, and the $\Gamma$-action can be extended to $\tilde{X}$. Let $\tilde{\rho}_{i}: \tilde{X} \rightarrow \tilde{X}_{i}$ be the quotient morphism of the $H_{i}$-action on $\tilde{X}$. For any nontrivial $f \in \mathbb{C}[\tilde{S}]$ whose zero locus contains $\tilde{S} \backslash S$ the $f$-localizations (i.e. the localizations with respect to the multiplicative system generated by $f$ ) of algebras $\mathbb{C}[\tilde{X}]$ and $\mathbb{C}[X]$ are isomorphic. Hence the similar localizations of $\mathbb{C}\left[\tilde{X}_{i}\right]$ and $\mathbb{C}\left[X_{i}\right]$ are isomorphic. This implies that the natural morphism $X_{i} \rightarrow \tilde{X}_{i}$ is an embedding (over $S \hookrightarrow \tilde{S}$ ) and we have the following commutative diagram

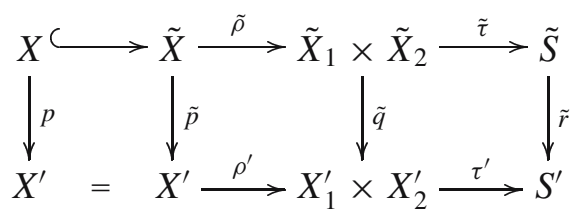

where $\tilde{\rho}=\left(\tilde{\rho}_{1}, \tilde{\rho}_{2}\right)$ extends $\rho=\left(\rho_{1}, \rho_{2}\right): X \rightarrow X_{1} \times X_{2}, \varrho^{\prime}=\tau^{\prime} \circ \rho^{\prime}$, and the morphisms $\tilde{\rho}, \tilde{q}, \tilde{r}$ are finite.

Set $Z$ (resp. $Z^{\prime}$, resp. $\tilde{Z}$ ) equal to the closure of $\rho(X)$ in $X_{1} \times X_{2}$ (resp. $\rho^{\prime}\left(X^{\prime}\right)$ in $X_{1}^{\prime} \times X_{2}^{\prime}$, resp. $\tilde{\rho}(\tilde{X})$ in $\left.\tilde{X}_{1} \times \tilde{X}_{2}\right)$. By Proposition $3.4 \rho: X \rightarrow Z$ is birational and hence $\tilde{\rho}: \tilde{X} \rightarrow \tilde{Z}$ is birational being the extension of $\rho$. By the claim in the proof of Lemma 3.6 morphism $\rho^{\prime}: X^{\prime} \rightarrow Z^{\prime}$ is birational. Note also that $\rho^{\prime}: X^{\prime} \rightarrow Z^{\prime}$ is quasi-finite (indeed, otherwise the commutative diagram implies that contrary to Proposition $3.4 \rho$ would not be quasi-finite because $\tilde{q}$ is finite and $p$ is surjective).

Suppose that $z^{\prime} \in Z^{\prime}$ and $s^{\prime}=\tau^{\prime}\left(z^{\prime}\right)$. Since $r$ is surjective one can choose $s \in S \subset \tilde{S}$ with $\tilde{r}(s)=s^{\prime}$. Take $z \in \tilde{q}^{-1}\left(z^{\prime}\right) \cap \rho(X)$ so that $\tilde{\tau}(z)=s$ (we can do this because the natural projection $X \rightarrow S$ is surjective). Hence $z^{\prime} \in \rho^{\prime}\left(X^{\prime}\right)$, i.e. $\rho^{\prime}$ is surjective. Furthermore, for any sequence $\left\{x_{i}^{\prime}\right\}$ of points in $X^{\prime}$ such that $\rho^{\prime}\left(x_{i}^{\prime}\right) \rightarrow z^{\prime}$ we can choose $x_{i} \in p^{-1}\left(x_{i}^{\prime}\right)$ so that $\rho\left(x_{i}\right) \rightarrow z$. Since morphism $\rho: X \rightarrow Z$ is finite by Proposition 3.4, one can suppose that the sequence $\left\{x_{i}\right\}$ is convergent to a point $x \in X$. Hence the sequence $\left\{x_{i}^{\prime}\right\}$ is convergent to $x^{\prime}=p(x)$ which means that $\rho^{\prime}: X^{\prime} \rightarrow Z^{\prime}$ is proper. Being also quasi-finite, this morphism is finite by Grothendieck's theorem. Now Proposition 3.4 yields the desired conclusion.

3.8. Remark. We do not know whether the assumption, that $\varrho^{\prime}$ and $r$ are surjective, is essential. Without this assumption the statement of Lemma 3.7 says only that $p$ is quasi-finite since one can put $S=X / / \Gamma$ and $S^{\prime}=X^{\prime} / / \Gamma$. However the surjectivity of $p$ may be sufficient for our purposes. Indeed, our aim is to check preservation of the algebraic density property under quasi-finite morphisms and there are examples of affine algebraic manifolds that are not homogeneous (and, therefore, have no algebraic density property [19]) but contain Zariski dense affine algebraic subvarieties with the algebraic density property. For instance, the hypersur- 
face in $\mathbb{C}_{x, y, z}^{3}$ given by $x y=z^{2}-1$ has the algebraic density [13] and it is not difficult to show that it is isomorphic to the complement to the line $x=z=1$ in the hypersurface in $\mathbb{C}^{3}$ given by $x(x-1) y=z^{2}-1$. The dual graph of a simple normal crossing completion of the latter hypersurface cannot be contracted to a zigzag in the terminology of [10] and, hence, this hypersurface is not even quasi-homogeneous by Gizatullin's theorem.

Recall that an étale neighborhood of a point $y$ of an algebraic variety $Y$ is an étale morphism $g: W \rightarrow Y$ whose image contains $y$.

3.9. Proposition. Let $Y$ be a normal affine algebraic variety equipped with a trivial $\Gamma$-action (where $\Gamma$ is from Lemma 3.6) and $r: X \rightarrow Y$ be a surjective $\Gamma$-equivariant morphism. Suppose that for any $y \in Y$ there exists an étale neighborhood $g: W \rightarrow Y$ such that the vector fields induced by $\delta_{1}$ and $\delta_{2}$ on the fibred product $X \times_{Y} W$ are compatible. Then $\delta_{1}$ and $\delta_{2}$ are compatible.

Proof. Set $Y_{1}=g(W)$. Then the restrictions $\delta_{1}^{1}$ and $\delta_{2}^{1}$ of $\delta_{1}$ and $\delta_{2}$ to $X_{1}=$ $r^{-1}\left(Y_{1}\right)$ are compatible by Lemma 3.7. Suppose that $\left\{Y_{i}\right\}$ is a finite cover of $Y$ by open sets similar to $Y_{1}$ and notation $X_{i}, \delta_{1}^{i}, \delta_{2}^{i}$ have also the similar meaning. Without loss of generality we can assume that $Y_{i}=Y \backslash f_{i}^{-1}(0)$ for some $f_{i} \in \mathbb{C}\left[Y_{i}\right] \subset \mathbb{C}\left[X_{i}\right]$. Let $I_{i} \subset \mathbb{C}\left[X_{i}\right]$ be the largest ideal contained in $\operatorname{Span}\left(\operatorname{Ker} \delta_{1}^{i} \cdot \operatorname{Ker} \delta_{2}^{i}\right)$ and $I$ be the largest ideal in $\mathbb{C}[X]$ whose $f_{i}$-localization is contained in $I_{i}$ for every $i$. In particular, $I$ is non-zero since each $I_{i}$ is such. Show that $I \subset \operatorname{Span}\left(\operatorname{Ker} \delta_{1} \cdot \operatorname{Ker} \delta_{2}\right)$.

Indeed, $f_{i} \in \mathbb{C}[Y] \subset \operatorname{Ker} \delta_{j}, j=1,2$. Hence for every $a \in I$ there exists $k_{i}$ such that $a f_{i}^{k_{i}}$ is in $\operatorname{Span}\left(\operatorname{Ker} \delta_{1} \cdot \operatorname{Ker} \delta_{2}\right)$. By Hilbert's Nullstellensatz there are regular functions $g_{i}$ on $Y$ such that $\sum_{i} f_{i}^{k_{i}} g_{i} \equiv 1$. Since $g_{i}$ is in the kernel of $\delta_{1}$ we see that $a \in \operatorname{Span}\left(\operatorname{Ker} \delta_{1} \cdot \operatorname{Ker} \delta_{2}\right)$ which concludes the proof.

3.10. Corollary. Let a linear algebraic group $G$ act algebraically on $X$ so that $X / / G$ is affine, the quotient morphism $X \rightarrow X / / G$ is surjective (which is always true when $G$ is reductive) and makes $X$ an étale $G$-principal bundle over $X / / G$. Suppose that $\Gamma$ (from Lemma 3.6) is an algebraic subgroup of $G$ and the actions of $H_{i}, i=1,2$ on $G$ induced by left multiplication generate compatible derivations on $\mathbb{C}[G]$. Let the induced $H_{i}$-actions on $X$ correspond to derivations $\delta_{i}$ on $\mathbb{C}[X]$. Then $\delta_{1}$ and $\delta_{2}$ are compatible.

Theorem 3. Let $G$ be a linear algebraic group whose connected component is different from a torus or $\mathbb{C}_{+}$. Then $G$ has the algebraic density property.

Proof. Since all components of $G$ are isomorphic as varieties we can suppose that $G$ is connected. Recall that the unipotent radical $R$ of $G$ is an algebraic subgroup of $G$ [4, p. 183]. By Mostow's theorem [14] (see also [4, p. 181]) $G$ contains a (Levi) maximal closed reductive algebraic subgroup $L$ (which is, in particular, affine) such that $G$ is the semi-direct product 
of $L$ and $R$, i.e. $G$ is isomorphic as affine variety to the product $R \times L$. In case $L$ is trivial $G=R \simeq \mathbb{C}^{n}, n \geq 2$ and we are done by Corollary 2.2. In the case of both $R$ and $L$ being nontrivial we are done by Corollary 2.8 with $R$ playing the role of $X_{1}$ and $L$ of $X_{2}$.

Thus it remains to cope with reductive groups $G$. Let $Z \simeq\left(\mathbb{C}^{*}\right)^{n}$ denote the center of $G$ and $S$ its semisimple part. First we suppose that $Z$ is nontrivial. The case when $G$ is isomorphic as group to the direct product $S \times Z$ can be handled as above by Corollary 2.8 with $S$ playing the role of $X_{1}$ and $Z$ of $X_{2}$. In particular, we have a finite set of pairs of compatible vector fields $\left\{\delta_{1}^{k}, \delta_{2}^{k}\right\}$ as in Theorem 2. Furthermore, one can suppose that the fields $\delta_{1}^{k}$ correspond to one parameter subgroups of $S$ isomorphic to $\mathbb{C}_{+}$and $\delta_{2}^{k}$ to one parameter subgroups of $Z$ isomorphic to $\mathbb{C}^{*}$. In the general case $G$ is the factor group of $S \times Z$ by a finite (central) normal subgroup $\Lambda$. Since $\Lambda$ is central the fields $\delta_{1}^{k}, \delta_{2}^{k}$ induce completely integrable vector fields $\tilde{\delta}_{1}^{k}, \tilde{\delta}_{2}^{k}$ on $G$ while $\tilde{\delta}_{2}^{k}\left(x_{0}\right)$ is a generating set for some $x_{0} \in G$. By Lemma 3.6 the pairs $\left\{\tilde{\delta}_{1}^{k}, \tilde{\delta}_{2}^{k}\right\}$ are compatible and the density property for $G$ follows again from Theorem 2.

It remains now to consider a semi-simple $G$ which can be assumed simply connected by Lemma 3.6. That is, it is a product of simple Lie groups and by Corollary 2.8 it suffices to consider the case when $G$ is simple. Such $G$ contains $S L_{2}(\mathbb{C})$ as a subgroup. The existence of two compatible vector fields $\delta_{1}$ and $\delta_{2}$ on $S L_{2}(\mathbb{C})$ implies their existence on $G$ by Corollary 3.10. Since a simple Lie group is tangentially semi-homogenous (see Example 2.10) the algebraic density property for $G$ follows again from Theorem 2.

\section{Codimension 2 case}

Motivation and notation. In this section $X$ will be a closed affine algebraic subvariety of $\mathbb{C}^{n}$ whose codimension $n-k$ is at least 2. By the Hartogs theorem any completely integrable algebraic (or holomorphic) vector field on $\mathbb{C}^{n} \backslash X$ extends to a similar vector field on $\mathbb{C}^{n}$ tangent to $X$. In particular, the Lie algebra generated by completely integrable algebraic (or holomorphic) vector fields contains only vector fields tangent to $X$, i.e. there is no density property for $\mathbb{C}^{n} \backslash X$. In general there is no also hope that this Lie algebra coincides with the Lie algebra of all algebraic vector fields tangent to $\mathrm{X}$, since this would imply density property for $X$ (and our $X$ maybe even not smooth!). Therefore, it is natural to study the Lie algebra Lie $_{\text {alg }}\left(\mathbb{C}^{n}, X\right)$ generated by completely integrable algebraic vector fields on $\mathbb{C}^{n}$ that vanishes on $X$. According to Forstnerič the best possible result to expect is that $\mathrm{Lie}_{\text {alg }}\left(\mathbb{C}^{n}, X\right)$ is equal to the Lie algebra of all algebraic vector fields vanishing on $X$. We use notation $\operatorname{AVF}_{I}\left(\mathbb{C}^{n}\right)$ for the latter algebra where $I \subset \mathbb{C}^{[n]}$ is the defining ideal of $X$ (more generally, for any affine algebraic variety $Y$ and an ideal $L \subset \mathbb{C}[Y]$ we denote by $\operatorname{AVF}_{L}(Y)$ the Lie algebra of vector fields whose coordinate functions are from $L$ ). If the 
above property holds then the geometric structure of algebraic vector fields vanishing on $X$ has the algebraic density property in the terminology of Varolin [19].

We will prove this property under some weak additional assumption in Theorem 6 and a very close result without any additional assumption in Theorem 4. Both results lead to a generalization of the main theorem of the Andersén-Lempert theory, allowing now to construct holomorphic automorphisms of $\mathbb{C}^{n}$ not only with control on compacts but with additional control on algebraic subvarieties of codimension at least 2.

4.1. Lemma. The group Aut $\left(\mathbb{C}^{n}, X\right)$ of algebraic automorphisms of $\mathbb{C}^{n}$ identical on $X$ acts transitively on $Z=\mathbb{C}^{n} \backslash X$ and, furthermore, for any $z \in Z$ the image of any vector $v \in T_{z} Z$ under the isotropy group $\operatorname{Aut}\left(\mathbb{C}^{n}, X\right)_{z}$ generates $T_{Z} Z$ (compare with Definition 2.1).

Proof. By a theorem of Gromov [11] and Winkelmann [20] $Z$ is homogenous. We will use the idea of their proof. More precisely, consider a general linear projection $p: \mathbb{C}^{n} \rightarrow \mathscr{H} \simeq \mathbb{C}^{n-1}$ and a non-zero constant vector field $v$ such that $p_{*}(v)=0$. Then $p(X)$ is a subvariety of codimension at least 1 in $\mathscr{H}$. For every regular function $h$ on $\mathscr{H}$ that vanishes on $p(X)$ the vector field $h v$ generates a $\mathbb{C}_{+}$-action on $Z$. Changing $\mathcal{H}$ we get a transitive action.

Consider a general point $z \in Z$ whose projection $z_{0} \in \mathcal{H}$ is not in $p(X)$. Suppose that $h$ has a simple zero at $z_{0}$. By the claim in the proof of Corollary 2.8 the $\mathbb{C}_{+}$-action generated by $h v$ acts on $T_{z} Z$ by the formula $w \rightarrow w+d h(w) v(w)$ where $d h$ is the differential of $h$ and $w \in T_{z} Z$. Since $v$ may be chosen as a general constant vector field on $\mathbb{C}^{n}$ we see that Aut $\left(\mathbb{C}^{n}, X\right)_{z}$ induces an irreducible representation on $T_{z} Z$ which implies the second statement.

Theorem 4. There is an ideal $L \subset \mathbb{C}^{[n]}$ whose radical is I such that $\operatorname{Lie}_{\text {alg }}\left(\mathbb{C}^{n}, X\right)$ contains $A V F_{L}\left(\mathbb{C}^{n}\right)$.

Proof. Suppose that $x_{1}, \ldots, x_{n}$ is a coordinate system, $p_{i}: \mathbb{C}^{n} \rightarrow \mathbb{C}^{n-1}$ is a projection to the coordinate hyperplane $\mathscr{H}_{i}=\left\{x_{i}=0\right\}$, and $h_{i}$ is a nonzero function on $\mathscr{H}_{i}$ that vanishes on $p_{i}(X)$. Set $\delta_{i}=\partial / \partial x_{i}$ and choose $f_{i} \in \operatorname{Ker} \delta_{i}$. Then $f_{i} h_{i} \delta_{i}$ is a completely integrable algebraic vector field on $\mathbb{C}^{n}$ that vanishes on $X$, i.e. it generates a $\mathbb{C}_{+}$-action on $Z$ (since the elements of this action are from $\left.\operatorname{Aut}\left(\mathbb{C}^{n}, X\right)\right)$. Then

$$
\left[f_{1} h_{1} \delta_{1}, x_{1} f_{2} h_{2} \delta_{2}\right]-\left[x_{1} f_{1} h_{1} \delta_{1}, f_{2} h_{2} \delta_{2}\right]=f_{1} f_{2} h_{1} h_{2} \delta_{2}
$$

belongs to $\mathrm{Lie}_{\text {alg }}\left(\mathbb{C}^{n}, X\right)$. Since $\operatorname{Ker} \delta_{1} \cdot \operatorname{Ker} \delta_{2}$ generates the ring of polynomials $\mathbb{C}^{[n]}$ as a vector space we see that $\operatorname{Lie}_{\text {alg }}\left(\mathbb{C}^{n}, X\right)$ contains all algebraic fields proportional to $\delta_{2}$ with coordinate functions in the principal ideal generated by $h_{1} h_{2}$. Since one can perturb $x_{2}$ (as a linear function) Lie alg $\left(\mathbb{C}^{n}, X\right.$ ) contains all algebraic vector fields whose coordinates are in some (non-zero) ideal $L$. Since $Z$ is homogenous under $\operatorname{Aut}\left(\mathbb{C}^{n}, X\right)$ arguing as in the proof of Theorem 1 one can suppose that the radical of $L$ is $I$. 
Though Theorem 4 does not give the algebraic density of the Lie algebra of algebraic vector fields vanishing on $X$ it implies already a strong approximation result generalizing the Andersén-Lempert theorem. We omit its proof since it repeats the arguments in [9] with minor modifications.

Theorem 5. Let $X$ be an algebraic subvariety of $\mathbb{C}^{n}$ of codimension at least 2 and $\Omega$ be an open set in $\mathbb{C}^{n}(n \geq 2)$. Let $\Phi:[0,1] \times \Omega \rightarrow \mathbb{C}^{n} \backslash X$ be a $\mathcal{C}^{2}$-map such that for every $t \in[0,1]$ the restriction $\Phi_{t}=\left.\Phi\right|_{(t \times \Omega)}$ is an injective holomorphic map onto a Runge domain $\Phi_{t}(\Omega)$ in $\mathbb{C}^{n}$. If $\Phi_{0}$ can be approximated on $\Omega$ by holomorphic automorphisms of $\mathbb{C}^{n}$ identical on $X$, then for every $t \in[0,1]$ the map $\Phi_{t}$ can be approximated on $\Omega$ by such automorphisms.

As a consequence we recover the result of Buzzard and Hubbard [3] answering Siu's question (see also [8], Prop. 1.4.).

4.2. Corollary. Any point $z$ in the complement of an algebraic subset $X$ of $\mathbb{C}^{n}$ of codimension at least 2 has a neighborhood $U$ in $\mathbb{C}^{n} \backslash X$ that is biholomorphic to $\mathbb{C}^{n}$ (such $U$ is called a Fatou-Bieberbach domain).

Proof. Following the standard scheme of Rudin and Rosay, choose a ball $\Omega \subset \mathbb{C}^{n} \backslash X$ around $z$ and consider $\Phi_{t}$ contracting this ball radially towards $z$. The resulting automorphism approximating $\Phi_{1}$ from Theorem 5 has an attracting fixed point near $z$ and $z$ is contained in the basin of attraction. This basin is a Fatou-Bieberbach domain and it does not meet $X$ since the automorphism is identical on $X$.

Though Theorem 4 is sufficient for the approximation purposes, let us be accurate and establish the algebraic density for algebraic vector fields vanishing on $X$ under an additional assumption.

4.3. Convention. We suppose further in this section that the dimension of the Zariski tangent space $T_{x} X$ is at most $n-1$ for every point $x \in X$.

4.4. Lemma. Lie algebra $\operatorname{Lie}_{\text {alg }}\left(\mathbb{C}^{n}, X\right)$ contains $A V F_{I^{2}}\left(\mathbb{C}^{n}\right)$.

Proof. It suffices to show that for every point $o \in \mathbb{C}^{n}$ there exists a Zariski neighborhood $V$ and a submodule $M_{V}$ from $\operatorname{Lie}_{\text {alg }}\left(\mathbb{C}^{n}, X\right)$ such that its localization to $V$ coincides with the localization of $\operatorname{AVF}_{I^{2}}\left(\mathbb{C}^{n}\right)$ to $V$. Indeed, because of quasi-compactness we can find a finite number of such open sets $V_{i}$ that cover $\mathbb{C}^{n}$. Hence the coherent sheaves generated by $\operatorname{AVF}_{I^{2}}\left(\mathbb{C}^{n}\right)$ and $\sum_{i} M_{V_{i}}$ coincide locally which implies that they have the same global sections over affine varieties by Serre's theorem B. In fact, it suffices to show that the localization of $M_{V}$ to $V$ contains all fields from the localization of $\operatorname{AVF}_{I^{2}}\left(\mathbb{C}^{n}\right)$ to $V$ that are proportional to some general constant vector field $\delta$ which is our aim now. By Theorem 4 it is also enough to consider $o \in X$ only. The construction of the desired neighborhood $V$ of $o$ starts with the following. 
Claim. For any point $o \in X, l \geq \max \left(k+1, \operatorname{dim} T_{o} X\right)($ where $\operatorname{dim} X=k)$, and a general linear projection $p: \mathbb{C}^{n} \rightarrow \mathscr{H} \simeq \mathbb{C}^{l}$ one can choose a projection $p_{0}: \mathbb{C}^{n} \rightarrow \mathcal{H}_{0} \simeq \mathbb{C}^{l-1}$ for which

(i) $p_{0}=\varrho \circ p$ where $\varrho: \mathscr{H} \rightarrow \mathscr{H}_{0}$ is a general linear projection, and

(ii) there exists $h \in \mathbb{C}\left[\mathscr{H}_{0}\right] \simeq \mathbb{C}^{[l-1]} \simeq \varrho^{*}\left(\mathbb{C}^{[l-1]}\right) \subset \mathbb{C}^{[l]}$ such that $h$ does not vanish at $p_{0}(o)$ and $\left.p\right|_{X \backslash(h \circ p)^{-1}(0)}: X \backslash(h \circ p)^{-1}(0) \rightarrow p(X) \backslash h^{-1}(0)$ is an isomorphism.

Since $p$ is general the condition on $l$ implies that $p$ is a local isomorphism in a neighborhood of $o$ and, furthermore, since $\varrho$ is also general then by Bertini's theorem $p_{0}^{-1}\left(p_{0}(o)\right)$ contains only smooth points of $X$ except, may be, for $o$, i.e. $p$ is a local isomorphism in a neighborhood of each of these points which implies the claim.

From now on let $l=n-1$. Choose a general coordinate system $\bar{x}=$ $\left(x_{1}, \ldots, x_{n}\right)$ on $\mathbb{C}^{n}$ such that $p(\bar{x})=\left(x_{2}, \ldots, x_{n}\right)$ and $p_{0}(\bar{x})=\left(x_{3}, \ldots, x_{n}\right)$, i.e. $h=h\left(x_{3}, \ldots, x_{n}\right)$. Set $V=\mathbb{C}^{n} \backslash h^{-1}(0)$.

Since $p(X) \cap V \simeq X \cap V$ we have $x_{1}=r / h^{s}$ where $r$ is a polynomial in $x_{2}, \ldots, x_{n}$ and $s \geq 0$. Set $v_{i}=\partial / \partial x_{i}$ for $i \neq 2$, and $v_{2}=h^{s} \partial / \partial x_{2}+$ $\left(\partial r / \partial x_{2}\right) \partial / \partial x_{1}$. Then each $v_{i}$ is a locally nilpotent derivation and Ker $v_{1}$ contains the defining ideal $I_{p}$ of $p(X)$ in $\mathbb{C}[\mathcal{H}] \simeq \mathbb{C}^{[n-1]} \simeq p^{*}\left(\mathbb{C}^{[n-1]}\right) \subset \mathbb{C}^{[n]}$. Furthermore, for $\xi=h^{s} x_{1}-r$ we have $\xi \in \operatorname{Ker} v_{2}$, and $\xi$ (resp. $x_{2}$ ) is of degree 1 with respect to $v_{1}$ (resp. $v_{2}$ ). This implies that for $f, g \in I_{p}$ the vector fields that appear in the Lie brackets below are completely integrable and vanish on $X$ :

$$
\begin{gathered}
{\left[f v_{1}, \xi g v_{1}\right]=h^{s} f g v_{1},\left[\xi v_{2}, x_{2} \xi v_{1}\right]-\left[x_{2} \xi v_{2}, \xi v_{1}\right]=h^{s} \xi^{2} v_{1}} \\
\text { and }\left[\xi v_{2}, x_{2} f v_{1}\right]-\left[x_{2} \xi v_{2}, f v_{1}\right]=h^{s} \xi f v_{1}
\end{gathered}
$$

The defining ideal of $X \cap V$ is generated by $\xi$ and elements of $I_{p}$. Since $h$ is invertible on $V$ and $v_{1}$ is a general constant vector field from the formulas before we see that the localization of $\mathrm{Lie}_{\text {alg }}(Z)$ to $V$ contains the localization of $\mathrm{AVF}_{I^{2}}\left(\mathbb{C}^{n}\right)$ which is the desired conclusion.

Theorem 6. Let $X$ be a closed algebraic subset of $\mathbb{C}^{n}$ of codimension at least 2 such that the Zariski tangent space $T_{x} X$ has dimension at most $n-1$ for any point $x \in X$. Then $\operatorname{Lie}_{a l g}\left(\mathbb{C}^{n}, X\right)$ is equal to $A V F_{I}\left(\mathbb{C}^{n}\right)$, i.e. the vector fields vanishing on $X$ have algebraic density property.

Proof. Similarly to the proof of Lemma 4.4, it suffices to show that for every point $o \in \mathbb{C}^{n}$ there exists a Zariski neighborhood $V$ and a submodule from Lie $_{\text {alg }}\left(\mathbb{C}^{n}, X\right)$ such that its localization $M$ to $V$ coincides with localization of $\operatorname{AVF}_{I}\left(\mathbb{C}^{n}\right)$ to $V$. By Theorem 4 it is enough to consider $o \in X$ and, furthermore, it suffices to show that this localization $M$ contains all elements of $\mathrm{AVF}_{I}\left(\mathbb{C}^{n}\right)$ proportional to some general constant vector field.

Let $v_{i}, p, I_{p}$, and $\xi$ have the same meaning as in the proof of Lemma 4.4. Choose $v_{1}$ as this constant vector field. Since $I$ is generated by $\xi$ and $I_{p}$ one needs to show that all fields of the form $\mu=\left(\xi g_{0}+\sum g_{i} f_{i}\right) \nu_{1}$ are 
contained in $M$ where $g_{0}, g_{i}$ are regular on $V$ and $f_{i} \in I_{p}$. Since $p$ yields an isomorphism between $p(X) \cap V$ and $X \cap V$ there are functions $e_{0}, e_{i}$ that do not depend on $x_{1}$ and such that $\left.e_{0}\right|_{X}=\left.g_{0}\right|_{X}$ and $\left.e_{i}\right|_{X}=\left.g_{i}\right|_{X}$. Then $\mu=\left(\xi e_{0}+\sum e_{i} f_{i}\right) \nu_{1}+a v_{1}$ where $a$ belongs to the localization of $I^{2}$ to $V$ (e.g. $\left.a=\xi\left(g_{0}-e_{0}\right)+\sum\left(g_{i}-e_{i}\right) f_{i}\right)$. Since the first summand in the last formula for $\mu$ is completely integrable we have the desired conclusion from Lemma 4.4.

4.5. Remark. (1) The authors believe that the condition $\operatorname{dim} T_{x} X \leq n-1$ in Theorem 6 is essential. As a potential counterexample one may try to take $X$ equal to polynomial curve in $\mathbb{C}^{3}$ with one singular point whose Zariski tangent space is 3-dimensional. More precisely, let $L$ be the Lie algebra generated by vector fields that vanish on $X$ and have form $f \sigma$ where $f \in \operatorname{Ker} \sigma$ and $\sigma$ is either locally nilpotent or semi-simple. Then we can show that $L$ does not coincide with $\operatorname{AVF}_{I}\left(\mathbb{C}^{3}\right)$ but we do not know whether $L=\operatorname{Lie}_{\text {alg }}\left(\mathbb{C}^{3}, X\right)$.

(2) In view of Theorem 6 the assumptions of Theorem 5 can be weakened in case of $\operatorname{dim} T_{x} X \leq n-1$ to the following extend: the assumption $\Phi_{t}(\Omega) \cap$ $X=\varnothing$ can be replaced by the assumption that $\Phi_{t}$ is identical on $\Phi_{t}(\Omega) \cap X$ for all $t$.

(3) The assumption of codimension at least 2 for $X$ cannot be removed, since the complement to a hypersurface in $\mathbb{C}^{n}$ can be of general type or even Kobayashi hyperbolic and, therefore, there is no nontrivial completely integrable holomorphic vector field vanishing on $X$. Also the assumption that $X$ is not just a holomorphic but an algebraic subvariety of $\mathbb{C}^{n}$ cannot be weakened. This follows from the fact that there are holomorphic embeddings of $\mathbb{C}$ into $\mathbb{C}^{n}$ (for any $n$ ) such that the group of holomorphic automorphisms of $\mathbb{C}^{n}$ identical on the image is trivial (e.g., see [5]).

\section{References}

1. Andersén, E.: Volume-preserving automorphisms of $\mathbb{C}^{n}$. Complex Variables, Theory Appl. 14(1-4), 223-235 (1990)

2. Andersén, E., Lempert, L.: On the group of holomorphic automorphisms of $\mathbb{C}^{n}$. Invent. Math. 110(2), 371-388 (1992)

3. Buzzard, G.T., Hubbard, J.H.: A Fatou-Bieberbach domain avoiding a neighborhood of a variety of codimension 2. Math. Ann. 316(4), 699-702 (2000)

4. Chevalley, C.: Théorie des groupes de Lie. Tome II. Groupes algébriques. Actual. Sci. Ind., vol. 1152. Hermann \& Cie., Paris (1951) (in French)

5. Derksen, H., Kutzschebauch, F., Winkelmann, J.: Subvarieties of $\mathbb{C}^{n}$ with nonextendable automorphisms. J. Reine Angew. Math. 508, 213-235 (1999)

6. Freudenburg, G.: Algebraic theory of locally nilpotent derivations. Invariant Theory and Algebraic Tranformation Groups VII. Encycl. Math. Sci., vol. 136, p. 261. Springer, Berlin Heidelberg New York (2006)

7. Forstnerič, F.: Interpolation by holomorphic automorphisms and embeddings in $\mathbb{C}^{n}$. J. Geom. Anal. 9(1), 93-117 (1999)

8. Forstnerič, F.: On complete intersections. Ann. Inst. Fourier 51(2), 497-512 (2001)

9. Forstnerič, F., Rosay, J.-P.: Approximation of biholomorphic mappings by automorphisms of $\mathbb{C}^{n}$. Invent. Math. 112(2), 323-349 (1993) 
10. Gizatullin, M.H.: I. Affine surfaces that are quasihomogeneous with respect to an algebraic group. Math. USSR Izv. 5, 754-769 (1971); II. Quasihomogeneous affine surfaces. Ibid. 1057-1081

11. Gromov, M.: Oka's principle for holomorphic sections of elliptic bundles. J. Am. Math. Soc. 2(4), 851-897 (1989)

12. Hartshorne, R.: Algebraic Geometry. Springer, New York Berlin Heidelberg London (1977)

13. Kaliman, S., Kutzschebauch, F.: Density property for hypersurfaces $u v=p(\bar{x})$. Math. Z. (to appear)

14. Mostow, G.D.: Fully reducible subgroups of algebraic groups. Am. J. Math. 78, 200-221 (1956)

15. Schwarz, G.: The topology of algebraic quotients. In: Topological Methods in Algebraic Transformation Groups, pp. 135-151. Birkhäuser, Boston Basel Berlin (1989)

16. Toth, A., Varolin, D.: Holomorphic diffeomorphisms of complex semisimple Lie groups. Invent. Math. 139(2), 351-369 (2000)

17. Toth, A., Varolin, D.: Holomorphic diffeomorphisms of semisimple homogenous spaces. Compos. Math. 142(5), 1308-1326 (2006)

18. Varolin, D.: The density property for complex manifolds and geometric structures. J. Geom. Anal. 11(1), 135-160 (2001)

19. Varolin, D.: The density property for complex manifolds and geometric structures. II. Int. J. Math. 11(6), 837-847 (2000)

20. Winkelmann, J.: On free holomorphic $\mathbb{C}^{*}$-actions on $\mathbb{C}^{n}$ and homogeneous Stein manifolds. Math. Ann. 286, 593-612 (1990)

21. Winkelmann, J.: Invariant rings and quasiaffine quotients. Math. Z. 244(1), 163-174 (2003) 